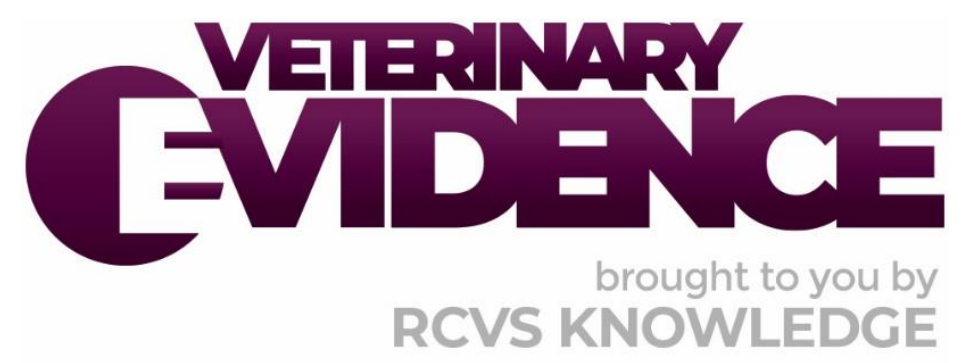

\title{
Does meniscal release confer similar clinical benefits to meniscal tear treatment when compared to meniscectomy?
}

\author{
A Knowledge Summary by
}

Ben Garland BVetMed CertAVP(GSAS) MRCVS ${ }^{1 *}$

${ }^{1}$ Christchurch Veterinary Referrals, Tuddenham Road Business park, Tuddenham Road, Ipswich,
Suffolk, IP4 3QN
${ }^{*}$ Corresponding Author (ben.veterinary@gmail.com)

ISSN: 2396-9776

Published: 21 Dec 2020

in: The Veterinary Evidence journal Vol 5, Issue 4

DOI: 10.18849/VE.V5I4.339

Reviewed by: Duncan Barnes (MA VetMB CertSAS DSAS(Orth) MRCVS) and Catrina Pennington (BVM\&S MRCVS)

Next Review Date: 02 Apr 2020 


\section{KNOWLEDGE SUMMARY}

\section{PICO question}

In adult dogs with naturally occurring medial meniscal tears concurrent to cranial cruciate ligament disease does meniscal release confer the same benefits in lameness resolution as meniscectomy?

\section{Clinical bottom line}

\section{Category of research question}

\section{Treatment}

\section{The number and type of study designs reviewed}

A single prospective cross-sectional study was reviewed, that fulfilled the criteria

\section{Strength of evidence}

None

\section{Outcomes reported}

Meniscal release, meniscectomy (partial, hemi- or complete), or the two combined performed for concurrent medial meniscal pathology at time of surgery for naturally occurring cranial cruciate ligament $(\mathrm{CCL})$ rupture resulted in an acceptable long-term outcome. Difference in outcome between the techniques was not reported

\section{Conclusion}

There is no evidence that meniscal release provides an equal or superior treatment option for medial meniscal injury treated at the time of surgery for $\mathrm{CCL}$ rupture when compared to meniscectomy. The study critically reviewed performed meniscal release via radial transection through the meniscotibial ligament, and therefore does not represent mid-body abaxial radial release. Neither is this summary appropriate for considering prophylactic meniscal release of the normal meniscus. In addition, the surgical treatments for cranial cruciate ligament rupture were either 'Tightrope' or tibial plateau levelling osteotomy (TPLO) procedures. Further studies are required to compare clinical outcome between meniscal release or meniscectomy for treatment of concurrent meniscal tears

\section{How to apply this evidence in practice}

The application of evidence into practice should take into account multiple factors, not limited to: individual clinical expertise, patient's circumstances and owners' values, country, location or clinic where you work, the individual case in front of you, the availability of therapies and resources.

Knowledge Summaries are a resource to help reinforce or inform decision making. They do not override the responsibility or judgement of the practitioner to do what is best for the animal in their care. 


\section{Clinical Scenario}

A 3-year-old male neutered Labrador retriever presents for investigation of a moderate right pelvic limb lameness localising to the right stifle with positive tibial compression and cranial drawer tests. You recommend radiographic investigation, exploratory arthrotomy, and surgical stabilisation to confirm and then treat suspected cranial cruciate ligament rupture. During an exploratory arthrotomy you confirm rupture of the cranial cruciate ligament and note a 'bucket handle' tear of the medial meniscus. You are aware that meniscal release is anecdotally used to treat this injury, but are uncertain this would provide a clinical outcome equal to partial meniscectomy.

\section{The evidence}

A single study was reviewed for this Knowledge Summary. There are no studies that directly compares the two treatment techniques investigated in this review making it impossible to perform systematic review or metaanalysis. To the author's knowledge, there are no prospective randomised or blinded studies that directly compare the outcome of meniscal release and meniscectomy surgeries for the treatment of meniscal injury at the time of surgical treatment of cranial cruciate ligament rupture. The evidence presented as part of this summary compares the outcome of the two techniques grouped together with no meniscal treatment. Meniscal treatment performed was selected based on intra-operative assessment and a surgeon's clinical judgement for each patient. Clinical outcomes were assessed at medium and long-term follow-up utilising subjective owner assessment. These results suggest that either surgical intervention for concurrent meniscal tears provides a high level of acceptable patient function when meniscal treatment was selected based upon surgeon assessment and clinical judgement. Criteria for surgical decision making has not yet been defined to determine if and when meniscal release may confer similar clinical benefits to meniscectomy techniques.

\section{Summary of the evidence}

\begin{tabular}{|c|c|}
\hline Population: & Adult dogs with naturally occurring cranial cruciate ligament disease \\
\hline Sample size: & 163 adult dogs, encompassing 223 stifles \\
\hline Intervention details: & $\begin{array}{l}\text { - Intra-articular stifle assessment, followed by surgical } \\
\text { stabilisation for cruciate insufficiency by one of: arthroscopy } \\
\text { and 'Tightrope' } 134 / 223(60 \%) \text {; arthroscopy and TLPO } \\
\text { 26/223 (12\%); or arthrotomy and TPLO 63/223 (28\%) } \\
\text { - Meniscectomy technique included 'partial' in 93/160 (85\%), } \\
\text { 'hemi' in 14/160 (13\% and 'complete' in 2/160 (2\%) }\end{array}$ \\
\hline Study design: & Prospective cross-sectional study \\
\hline Outcome studied: & $\begin{array}{l}\text { Incidence and type of meniscal pathology, and the effect of meniscal } \\
\text { pathology and its overall treatment on outcome in dogs managed } \\
\text { for cranial cruciate ligament rupture. } \\
\text { Medium (6-12 months) and long-term (>1 year) functional outcome } \\
\text { as assessed by owner questioning of: } \\
\text { - function } \\
\text { - pain } \\
\text { - postoperative complications } \\
\text { - current use of non-steroidal anti-inflammatory drugs or } \\
\text { other analgesics (associated with cranial cruciate ligament } \\
\text { disease). } \\
\text { Based on the results outcome was graded 'full', 'acceptable', or } \\
\text { 'unacceptable'. }\end{array}$ \\
\hline
\end{tabular}




\begin{tabular}{|c|c|}
\hline & $\begin{array}{l}\text { These were then grouped as successful (full and acceptable) or } \\
\text { unsuccessful (unacceptable) outcome. }\end{array}$ \\
\hline $\begin{array}{l}\text { Main findings: } \\
\text { (relevant to PICO question): }\end{array}$ & $\begin{array}{l}\text { - Of } 223 \text { cases, } 160 \text { ( } 72 \% \text { ) had concurrent meniscal tear } \\
\text { diagnosed at the time of inspection and stabilisation } \\
\text { surgery. Of these } 159 \text { were of the medial meniscus. } \\
\text { - All tears were treated with either meniscectomy } 109 / 160 \\
(68 \%) \text {, meniscal release (caudal) } 81 / 160 \text { ( } 51 \%) \text {, or a } \\
\text { combination of both } 30 / 160(19 \%) \text {. } \\
\text { Overall } 51 / 160 \text { ( } 32 \%) \text { meniscal release, } 79 / 160(49 \%) \\
\text { meniscectomy, } 30 / 160 \text { ( } 19 \% \text { ) both. (This had to be } \\
\text { extrapolated from the data presented). } \\
\text { - At medium term follow-up ( } 6-12 \text { months): owners of } 94 / 163 \\
\text { dogs contacted, } 68 \text { dogs had concurrent tears treated, of } \\
\text { which } 65 \text { ( } 96 \%) \text { had successful outcome } \\
\text { - At long-term follow-up (>1 year): owners of } 87 / 160 \text { dogs } \\
\text { contacted, } 62 \text { dogs had concurrent tears treated of which } 60 \\
\text { had a successful outcome (97\%) }\end{array}$ \\
\hline Limitations: & $\begin{array}{l}\text { - Outcome assessed meniscal intervention overall and does } \\
\text { not differentiate between treatment types } \\
\text { - Meniscectomy included partial, hemi-, or complete } \\
\text { - } \quad \text { Comparison and outcome were not grouped based on type } \\
\text { - Meniscal intervention was based on surgeon preference, } \\
\text { with surgeon number and skill level not clearly defined } \\
\text { - Meniscal treatment elected is not stated to be based on } \\
\text { - } \quad \text { Concurrent surgery for CCL rupture was not randomised nor } \\
\text { its impact on outcome assessed with respect to meniscal } \\
\text { surgery } \\
\text { - Population of dogs limited to patients referred to a single } \\
\text { referral centre } \\
\text { - Outcome was owner centric, subjectively assessed, and } \\
\text { grouped into broad categories } \\
\text { - Non-blinded } \\
\text { - } \text { Con-randomised } \\
\text { are not stated } \\
\text { - No statistical analysis is performed to consider the } \\
\text { significance of outcome achieved } \\
\text { A large proportion of treated dogs were lost to follow-up at } \\
\text { both 6-12 months and }>1 \text { year }\end{array}$ \\
\hline
\end{tabular}

\section{Appraisal, application and reflection}

Only a single study was identified from this literature review where meniscal release was used to treat meniscal tears identified at the time of surgery for cranial cruciate ligament rupture.

The study identified was observational, of prospective cross-sectional design, utilising a prospectively maintained database to retrospectively obtain data. Importantly, this study design was not intended to directly compare the effect of meniscal release with meniscectomy in a cohort of dogs. Instead, meniscal release and meniscectomy (partial, hemi-, or complete) were grouped together when considering clinical outcome. Whilst 
this study provides evidence in relation to disease prevalence (as per the intentions of the study's authors), when considering the impact of meniscal surgical techniques overall the evidence would be considered weak [Class III] (Aragon \& Budsberg, 2005; and Dean, 2013). Furthermore, when considering the impact between meniscal interventions this study does not provide evidence that meniscal release confers the same clinical outcome as meniscectomy.

When considering this study in the context of the clinical question posed several confounding factors can be identified. Randomisation was not performed for either meniscal intervention or the surgical procedure used to treat $\mathrm{CCL}$ rupture. As such bias is introduced by the surgeon, when considering outcome against intervention performed. Surgeon experience was recorded; however, this was not stratified to the differing interventions with surgeon skill or confidence open to influencing outcome. Meniscal assessment method was not standardised, with arthrotomy or arthroscopy used. This could impact the detection sensitivity of meniscal pathology between treatment groups (Pozzi et al., 2008a; and Plesman et al., 2012). As undiagnosed meniscal pathology is recognised as a potential factor for suboptimal clinical outcome following surgery for cranial cruciate ligament rupture, utilising techniques of different sensitivity could confound outcome (Fitzpatrick \& Solano, 2010; and Thieman et al., 2006). Similar rates of subsequent meniscal tears were obtained between all treatment groups reported (range 6.3-7.7\%) and are within previously reported values (McReady \& Ness, 2016a). Meniscal treatment selection was not linked to the type of pathology encountered, which may have unrecognised implications upon outcome given the breadth of concurrent medial meniscal pathology diagnosed (Cook \& Pozzi, 2010). This could have clinical significance if outcome is linked to pathology encountered and surgical technique performed. The cohort of dogs included in this study consisted exclusively of a wide range of individuals referred to a single centre. Patients factors such as bodyweight, body condition score, and co-morbidities are reported but not linked to outcome obtained. When addressing outcome, medium and long-term follow-up was available for a proportion of patients. A considerable number were lost to follow-up, with $42 \%$ and $39 \%$ of all 223 cases achieving medium and long-term follow-up respectively. Outcome was based subjectively on owner assessment without the use of objective measures or a validated metrology instrument (McColl et al., 2002; and Muller et al., 2016). Outcomes were broadly grouped into 'full function','acceptable function', and 'unacceptable function' based on previously defined criteria in veterinary orthopaedics (Cook et al 2010). A successful outcome was considered to be either 'full' or 'acceptable' function, therefore successful outcome should be regarded cautiously. Statistical analysis to consider the significance of findings presented are not given within the study. Whilst confidence intervals were discussed in the methodology, these were not expressed further in the results, nor are power calculations presented to determine group sizes. Most importantly in relation to the clinical question, outcome was not separated between meniscal interventions or status at primary examination but instead grouped together. Therefore, when considering this, regardless of limitations this paper only demonstrates successful outcome for the reported meniscal interventions for concurrent tears as a whole. As such, in relation to the clinical question posed here, this Knowledge Summary is unable to provide evidence that meniscal release confers the same benefits as meniscectomy in the clinical outcome obtained.

The amount of available literature to consider this question is sparse, with very limited reference to meniscal release as a therapeutic technique for meniscal pathology. Meniscectomy procedures are the most widely adopted technique for meniscal pathology treatment (Cook \& Pozzi, 2010), with meniscal repair methods described for select meniscal tears (Rovesti et al., 2018, and Thieman et al., 2010). Notably, the most robust evidence in this field is a recent meta-analysis by McCready \& Ness (2016a and b) investigating both cranial cruciate ligament treatment surgery and meniscal pathology diagnosis and treatment; however meniscal release as a treatment for concurrent tears did not feature in their analysis.

Anecdotally, some surgeons utilise meniscal release as their preferred treatment option for concurrent meniscal tears. Meniscal release is relatively less technically demanding than meniscectomy, is faster, and can be performed via blind or minimally invasive methods (Austin et al., 2007). Given the commonality of cranial cruciate ligament disease in the canine population (Wilke et al., 2005) with reported rates of 10-77\% concurrent meniscal tears (Cook \& Pozzi, 2010), successful meniscal treatment via the least invasive method 
possible may prove beneficial for patients and surgical efficiency considering the similar biomechanics reported between techniques (Pozzi et al., 2008b, 2010a, and 2010b). However, it is therefore important to determine if meniscal release confers the same well documented benefits to patients in lameness resolution as meniscectomy procedures before considering this an evidence based surgical technique. From this Knowledge Summary there is no evidence to support meniscal release as equal to meniscectomy in clinical outcome achieved when treating concurrently diagnosed medial meniscal tears at the time of index surgery for naturally occurring cranial cruciate ligament rupture. Other significant limitations include the unknown effect of the index surgery used to treat $\mathrm{CCL}$ rupture on the efficacy of meniscal treatment. Furthermore, the meniscal release utilised was exclusively axial (also known as 'caudal' or 'menisco-tibial') which should be borne in mind when considering clinical application. A well designed, standardised prospective randomised control study would be required with objective long-term outcome measures to investigate this clinical question.

\section{Methodology Section}

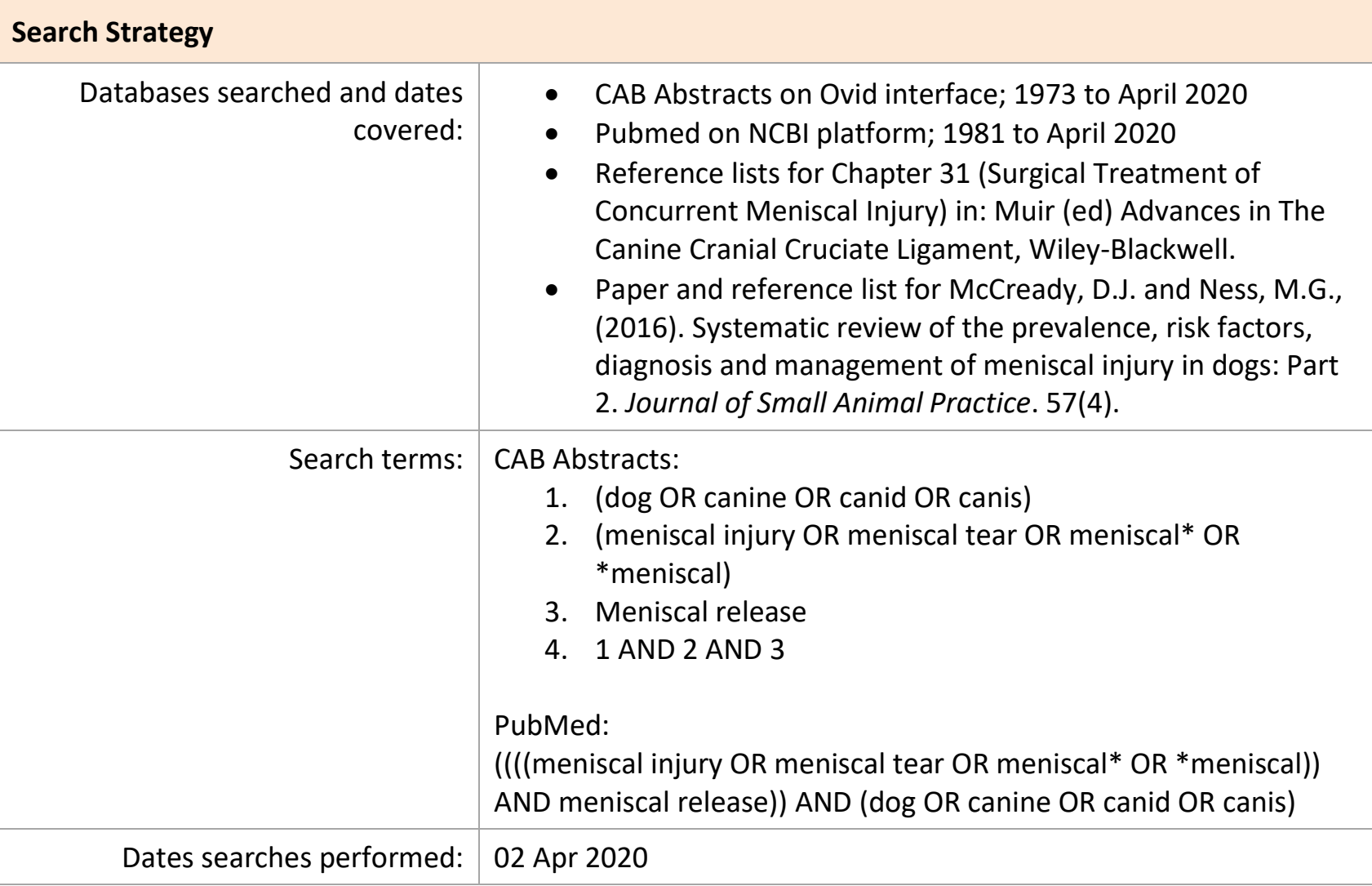

\begin{tabular}{|r|l|}
\hline \multicolumn{2}{|l|}{ Exclusion / Inclusion Criteria } \\
$\qquad \begin{aligned} \text { Exclusion: } \\
\text { Inclusion: }\end{aligned}$ & $\begin{array}{l}\text { Experimental studies; where meniscal release was used only to treat } \\
\text { a normal meniscus; where meniscal inspection was not performed; } \\
\text { ex vivo or biomechanical studies; papers not available in English; } \\
\text { papers that could not be accessed }\end{array}$ \\
\hline $\begin{array}{l}\text { Where meniscal release was used as a therapeutic treatment for } \\
\text { meniscal tears }\end{array}$ \\
\hline
\end{tabular}




\begin{tabular}{|c|c|c|c|c|c|c|}
\hline \multicolumn{7}{|c|}{ Search Outcome } \\
\hline Database & $\begin{array}{c}\text { Number } \\
\text { of } \\
\text { results }\end{array}$ & $\begin{array}{l}\text { Excluded - } \\
\text { after } \\
\text { reading } \\
\text { title }\end{array}$ & $\begin{array}{c}\text { Excluded - after } \\
\text { reading } \\
\text { abstract }\end{array}$ & $\begin{array}{l}\text { Excluded - after } \\
\text { reading } \\
\text { materials and } \\
\text { methods }\end{array}$ & $\begin{array}{l}\text { Excluded - } \\
\text { paper could } \\
\text { not be } \\
\text { accessed or } \\
\text { was not in } \\
\text { English }\end{array}$ & $\begin{array}{l}\text { Total } \\
\text { relevant } \\
\text { papers }\end{array}$ \\
\hline $\begin{array}{l}\text { CAB } \\
\text { Abstracts }\end{array}$ & 36 & 6 & 10 & 13 & 6 & 1 \\
\hline PubMed & 33 & 12 & 12 & 8 & 0 & 1 \\
\hline Muir & 15 & 6 & 4 & 2 & 3 & 0 \\
\hline $\begin{array}{l}\text { McCready \& } \\
\text { Ness }\end{array}$ & 104 & 63 & 13 & 25 & 2 & 1 \\
\hline \multicolumn{6}{|c|}{ Total relevant papers when duplicates removed } & 1 \\
\hline
\end{tabular}

\section{CONFLICT OF INTEREST}

The author declares no conflicts of interest.

The author would like to thank Ms Clare Boulton at the RCVS Knowledge Library service for her assistance in searching the literature.

\section{REFERENCES}

1. Aragon, C. and Budsberg, S. (2005). Applications of evidence-based medicine: cranial cruciate ligament injury repair in the dog. Veterinary Surgery. 34, 93-8. DOI: http://dx.doi.org/10.1111/i.1532950X.2005.00016.x

2. Austin, B., Montgomery, R.D., Wright, J., Bellah, J.R. and Tonks, C. (2007). Evaluation of three approaches to meniscal release. Veterinary and Comparative Orthopaedics and Traumatology. 20(2), 92-97. DOI: http://dx.doi.org/10.1160/vcot-06-04-0033

3. Cook, J.L., Evans, R., Conzemius, M.G., Lascelles, B.D.X., Mcllwraith, C.W., Pozzi, A., Clegg, P., Innes, J., Schulz, K., Houlton, J., Fortier, L., Cross, A.R., Hayashi, K., Kapatkin, A., Brown, D.C. and Stewart, A. (2010). Proposed Definitions and Criteria for Reporting Time Frame, Outcome, and Complications For Clinical Orthopedic Studies in Veterinary Medicine. Veterinary Surgery. 39, 905-908.

DOI: https://doi.org/10.1111/j.1532-950X.2010.00763.x

4. Cook, J.L. and Pozzi, A. (2010). Surgical treatment of concurrent meniscal injury. In: Muir, P. ed., Advances in The Canine Cranial Cruciate Ligament $1^{\text {st }}$ Edition. Wiley-Blackwell, 217-222.

5. Dean, R. (2013). How to read a paper and appraise the evidence. In Practice. 35, 282-285.

DOI: http://dx.doi.org/10.1136/inp.f1760 
6. Fitzpatrick, N. and Solano, M.A. (2010). Predictive Variables for Complications after TPLO with Stifle Inspection by Arthrotomy in 1000 Consecutive Dogs. Veterinary Surgery. 39, 460-474.

DOI: https://doi.org/10.1111/j.1532-950X.2010.00663.x

7. McColl, E., Jacoby, A., Thomas, L., Soutter, J., Bamford, C., Steen, N., Thomas, R., Harvey, E., Garratt, A. and Bond, J. (2002). Design and use of questionnaires: a review of best practice applicable to surveys of health service staff and patients. Health Technology Assessment. 5(31).

DOI: https://doi.org/10.3310/hta5310

8. McCready, D.J. and Ness, M.G. (2016a). Diagnosis and management of meniscal injury in dogs with cranial cruciate ligament rupture: a systematic literature review. Journal of Small Animal Practice. 57(2), 59-66. DOI: https://doi.org/10.1111/jsap.12433

9. McCready, D.J. and Ness, M.G. (2016b). Systematic review of the prevalence, risk factors, diagnosis and management of meniscal injury in dogs: Part 2. Journal of Small Animal Practice. 57(4), 194-204. DOI: https://doi.org/10.1111/jsap.12462

10. Muller, C., Gaines, B., Gruen, M., Case, B., Arrufat, K., Innes, J. and Lascelles, B.D.X. (2016). Evaluation of Clinical Metrology Instrument in Dogs with Osteoarthritis. Journal of Veterinary Internal Medicine. 30(3), 836-846. DOI: https://doi.org/10.1111/jvim.13923

11. Plesman, R., Gilbert, P. and Campbell, J. (2013). Detection of meniscal tears by arthroscopy and arthrotomy in dogs with cranial cruciate ligament rupture: a retrospective, cohort study. Veterinary and Comparative Orthopaedics and Traumatology. 26(1), 42-46.

DOI: http://dx.doi.org/10.3415/VCOT-11-10-0153

12. Pozzi, A., Hildreth, B.E. and Rajala-Schultz, P.J. (2008a). Comparison of Arthroscopy and Arthrotomy for Diagnosis of Medial Meniscal Pathology: An Ex Vivo Study. Veterinary Surgery. 37, 749-755. DOI: https://dx.doi.org/10.1111/j.1532-950x.2008.00442.x

13. Pozzi, A., Litsky, A.S., Field, J., Apelt, D., Meadows, C. and Johnson, K.A. (2008b). Pressure distributions on the medial tibial plateau after medial meniscal surgery and tibial plateau levelling osteotomy in dogs. Veterinary and Comparative Orthopaedics and Traumatology. 21, 8-14.

DOI: https://doi.org/10.3415/VCOT-06-12-0099

14. Pozzi, A., Kim, S. E. and Lewis, D.D. (2010a). Effect of transection of the caudal menisco-tibial ligament on medial femorotibial contact mechanics. Veterinary Surgery. 39, 489-495.

DOI: https://doi.org/10.1111/j.1532-950X.2010.00662.x

15. Pozzi, A., Tonks, C.A. and Ling, H.Y. (2010b). Femorotibial contact mechanics and meniscal strain after serial meniscectomy. Veterinary Surgery. 39, 482-488. DOI: https://doi.org/10.1111/j.1532950X.2010.00668.x

16. Rovesti, G.L., Devesa, V., Bertorelli, L. and Rodriguez-Quiros, J. (2018). Facilitation of arthroscopic visualization and treatment of meniscal tears using a stifle joint distractor in the dog. BMC Veterinary Research. 14(1), 212. DOI: https://doi.org/10.1186/s12917-018-1534-9

17. Ritzo, M.E., Ritzo, B.A., Siddens, A.D., Summerlott, S. and Cook, J.L. (2014). Incidence and type of meniscal injury and associated long-term clinical outcomes in dogs treated surgically for cranial cruciate ligament disease. Veterinary Surgery. 43(8), 952-958. DOI: https://doi.org/10.1111/i.1532950X.2014.12220.x

18. Thieman, K.M., Tomlinson, J.L., Fox, D.B., Cook, C. and Cook, J.L. (2006). Effect of Meniscal Release on Rate of Subsequent Meniscal Tears and Owner-Assessed Outcome in Dogs with Cruciate Disease Treated with Tibial Plateau Leveling Osteotomy. Veterinary Surgery. 35, 705-710.

DOI: https://doi.org/10.1111/j.1532-950X.2006.00214.x

19. Thieman, K.M., Pozzi, A., Ling, H.-Y. and Lewis, D. (2010). Comparison of Contact Mechanics of Three Meniscal Repair Techniques and Partial Meniscectomy in Cadaveric Dog Stifles. Veterinary Surgery. 39, 355-362. DOI: https://doi.org/10.1111/j.1532-950X.2010.00661.x

20. Wilke, V.L, Robinson, D.A., Evans, R.B., Rothschild, M.F. and Conzemius, M.G. (2005). Estimate of the annual economic impact of treatment of cranial cruciate ligament injury in dogs in the United States. Journal of the American Veterinary Medication Association. 227, 1604-1607. DOI: https://doi.org/10.2460/javma.2005.227.1604 


\section{EVIIDEFeE

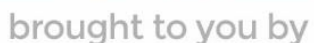 \\ RCVS KNOWLEDGE}

\section{Intellectual Property Rights}

Authors of Knowledge Summaries submitted to RCVS Knowledge for publication will retain copyright in their work, and will be required to grant RCVS Knowledge a non-exclusive license of the rights of copyright in the materials including but not limited to the right to publish, re-

publish, transmit, sell, distribute and otherwise use the materials in all languages and all media throughout the world, and to license or permit others to do so.

\section{Disclaimer}

Knowledge Summaries are a peer-reviewed article type which aims to answer a clinical question based on the best available current evidence. It does not override the responsibility

of the practitioner. Informed decisions should be made by considering such factors as individual clinical expertise and judgement along with patient's circumstances and owners' values. Knowledge Summaries are a resource to help inform and any opinions expressed within the Knowledge Summaries are the author's own and do not necessarily reflect the view of the RCVS Knowledge. Authors are responsible for the accuracy of the content. While the

Editor and Publisher believe that all content herein are in accord with current recommendations and practice at the time of publication, they accept no legal responsibility

for any errors or omissions, and make no warranty, express or implied, with respect to material contained within.

For further information please refer to our Terms of Use.

RCVS Knowledge is the independent charity associated with the Royal College of Veterinary Surgeons (RCVS). Our ambition is to become a global intermediary for evidence based veterinary knowledge by providing access to information that is of immediate value to practicing veterinary professionals and directly contributes to evidence based clinical decision-making.

https://www.veterinaryevidence.org/

RCVS Knowledge is a registered Charity No. 230886.

Registered as a Company limited by guarantee in England and Wales No. 598443.

Registered Office: Belgravia House, 62-64 Horseferry Road, London SW1P 2AF

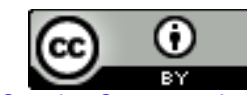

This work is licensed under a Creative Commons Attribution 4.0 International License 